\title{
NEW COMPOSITIONS OF FLUOROINDATE GLASSES WITH HIGHER CHEMICAL RESISTANCE
}

\section{B. J. Costa*}

IFSC - USP - CP 369 - 13560-250 - São Carlos - SP

A. Soufiane

Full Spectrum, Inc. - 2515 Elwood Drive - Unit 108 - Ames - Iowa - 50010 - USA

Y. Messaddeq

IQ - UNESP - CP 355 - 14800-970 - Araraquara - SP

Recebido em 2/12/96; aceito em 22/4/97

\begin{abstract}
The chemical resistance of fluoride glasses in aqueous solution is a very important factor concerning the technological applications of these materials. In this paper, glasses in the systems In-Ba$\mathrm{Mg}$ and In-Ba-Zn-Sr-Mg were water leachead at $80^{\circ} \mathrm{C}$ showing surface degradation after 72 hours of leaching. The extent of such degradation is determined by the solubility and the concentration of the elemental fluorides that constitute the glasses. The formation of a layer of crystallized phases on the surface of the samples was observed. Small weight losses were registered and the absence of water on the glass matrix after the attack suggested that the use of $\mathrm{MgF}_{2}$ in the systems studied can lead to better results against moisture corrosion when compared to other fluoride glasses such as the fluorozirconates.
\end{abstract}

Keywords: glass; fluoroindate; water; resistance.

\section{INTRODUCTION}

Fluoroindate glasses are being developed and studied in many laboratories for use as infrared transmitting optical components such as fiber waveguides, laser windows and IR lenses, due to their extended transparency in the mid-infrared spectral range ${ }^{1}$. Since these glasses are likely to come in contact with water at some point during their use, it is desirable to investigate their chemical resistance in aqueous solutions. In this work, it is described the effect of $\mathrm{MgF}_{2}$ on new compositions of the binary system In-Ba and the system In-Ba-Zn-Sr, concerning their chemical durability in distilled water.

\section{EXPERIMENTAL PROCEDURE}

The fluoroindate glass samples studied in this paper were prepared according to the method of ammonium bifluoride described by Costa and Messaddeq ${ }^{2}$. Compositions are given in table 1 .

Table 1. Compositions of fluoroindate glasses prepared in this work.

\begin{tabular}{cccccc}
\hline Glass & \multicolumn{5}{c}{ Composition $(\mathrm{mol} \%)$} \\
\hline & In & $\mathrm{Ba}$ & $\mathrm{Sr}$ & $\mathrm{Zn}$ & $\mathrm{Mg}$ \\
1 & 50 & 40 & - & - & 10 \\
2 & 47.5 & 37.5 & - & - & 15 \\
3 & 40 & 15 & 20 & 20 & 5 \\
4 & 40 & 10 & 20 & 20 & 10 \\
5 & 33.8 & 12.6 & 18.9 & 18.9 & 15.8 \\
\hline
\end{tabular}

Glasses of initial weight around $2 \mathrm{~g}$ (approximately $20 \mathrm{~mm}$ long, $10 \mathrm{~mm}$ wide and $3 \mathrm{~mm}$ thick) were water leached at $80^{\circ} \mathrm{C}$ in a Soxhlet apparatus for 72 hours. After the attack, the samples were dried in air and changes in weight were determined by

* Permanent address: Tecpar - CP 357 - 80001-970 - Curitiba - PR
$\left(\Delta \mathrm{m} / \mathrm{m}_{\mathrm{A}}\right) \cdot 100$, where $\mathrm{m}_{\mathrm{A}}$ is the initial weight of the glass sample. The corrosion product on the glass surface was examined by Scanning Electron Microscopy (SEM), Energy-Dispersive XRay (EDX) and X-Ray Diffraction (XRD) techniques.

\section{RESULTS AND DISCUSSION}

According to Poulain ${ }^{3}$ and Soufiane ${ }^{4}$, up to $30 \% \mathrm{MgF}_{2}$ could be incorporated in various fluoride glasses, ranging from fluoroaluminates to fluoroindates. As a consequence, an improvement in the practical properties of these glasses may be expected, specially the resistance to moisture. The results of the aqueous leaching of glass samples of both systems investigated are presented in table 2 .

Table 2. Weight losses of $\mathrm{InF}_{3}$ glasses after water leaching.

\begin{tabular}{cc}
\hline Glass & Weight loss $(\%)$ \\
\hline 1 & 0.91 \\
2 & 0.89 \\
3 & 0.27 \\
4 & 0.55 \\
5 & 0.31
\end{tabular}

The weight losses of the fluoroindate glasses studied are smaller than those obtained by Frischat and Overbeck ${ }^{5}$ for fluorozirconate glasses after water attack at $80^{\circ} \mathrm{C}$ in a Soxhlet apparatus for only 1 hour of leaching according to table 3 .

Table 3. Weight losses of $\mathrm{ZrF}_{4}$ glasses after water leaching according to Frischat and Overbeck ${ }^{5}$.

\begin{tabular}{cc}
\hline Glass & Weight loss $(\%)$ \\
\hline 1 & 1.16 \\
2 & 2.24 \\
3 & 0.63 \\
4 & 0.98
\end{tabular}


The changes in weight described, as well as those reported in table 3, were determined without the removal of the surface corroded layer of the glasses. Recently, da Silva ${ }^{6-7}$ studied the water corrosion of fluoroindate glasses of several systems (IBZS, IBZSNGd, and IBZSNGa), at room temperature for 72 hours, without $\mathrm{MgF}_{2}$ and observed that these glasses are rapidly attacked in aqueous medium, resulting the formation of a surface film with a large amount of adsorbed water after a few minutes of testing. No weight changes were reported but vibrational IR spectroscopy revealed the presence of water adsorbed and/or incorporated to the glass matrix. In this case, the corrosion mechanism proposed consists of an initial step when the matrix is dissolved, followed by the formation of sites and the adsorption of $\mathrm{M}-\mathrm{OH}$ compounds in the glass surface. The experiments previously discussed suggest a better water corrosion resistance of magnesium-doped fluoroindate glasses, particularly those samples of the In-Zn-Sr-Ba system, when compared to fluorozirconate and undoped fluoroindate glasses.

The exposure of the fluoroindate glasses to liquid water, under the chosen conditions, resulted in formation of an opaque surface layer in all samples investigated. The inspection of the corroded glasses by SEM, showed the presence of precipitated crystal deposits over the entire surface of the glasses. Cubiclike crystals can be observed in the micrograph of figure 1 . The technique of EDX was used to investigate the chemical nature of the crystal layer deposited on the glass surface. The results indicated that indium was the only glass component leached/dissolved and segregated at the glass surface. An XRD study of such surface crystals helped to identify the main phases $\mathrm{In}(\mathrm{OH})_{3}, \gamma-\mathrm{InF}_{3}$ and $\mathrm{In}_{2} \mathrm{O}_{3}$ as according to figure 2 .

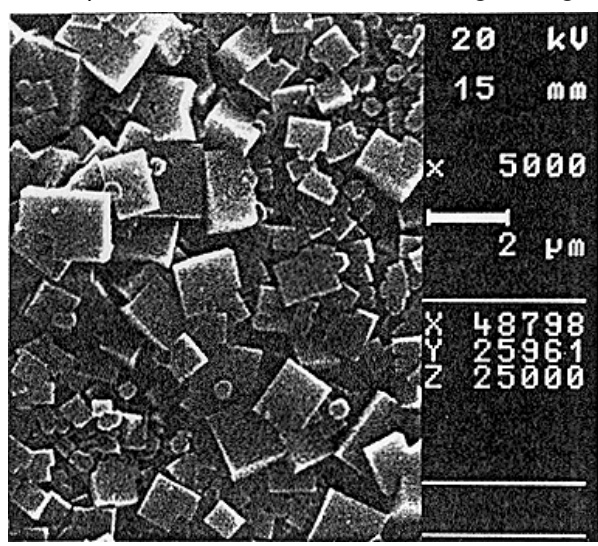

Figure 1. Crystal deposits formed during water leaching of fluoroindate glasses.

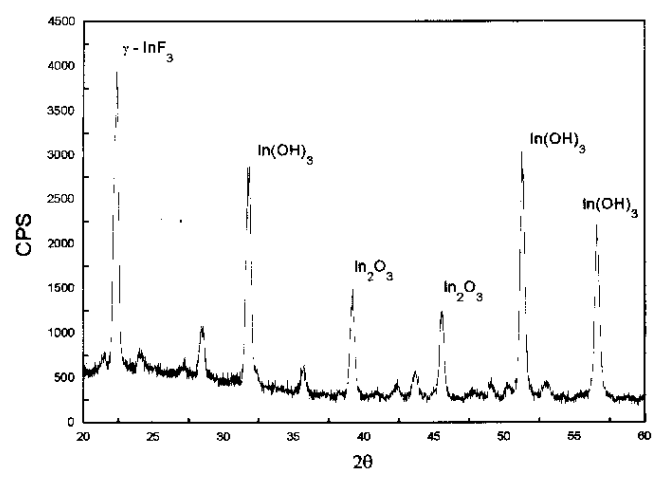

Figure 2. XRD pattern of crystal deposits on glass surface after water attack.

In order to verify if cations $\mathrm{Zn}, \mathrm{Ba}, \mathrm{Sr}$ and $\mathrm{Mg}$ were leached or not from the glasses tested, the leaching solutions are presently being analyzed for those glass components. The presence of fluoride and indium in the solutions is also under investigation. The results will be reported in a future paper.
The IR transmission spectrum of figure 3 was obtained after water attack, removal of the corroded surface layer and repolishing of one of the fluoroindate glasses tested. In such spectrum, it cannot be observed the presence of water that produces a characteristic strong absorption peak at $3400 \mathrm{~cm}^{-1}$, due to stretching vibrations of $\mathrm{O}-\mathrm{H}$ bonding, and a medium-weak absorption peak around 1640 $\mathrm{cm}^{-1}$ attributed to $\mathrm{O}-\mathrm{H}$ bending vibrations ${ }^{8}$. This indicates that corrosion in the $\mathrm{Mg}$-doped fluoroindate glasses was limited to the thin surface layer (estimated to be around $0,5 \mu \mathrm{m}$ thick) observed. As the glass matrix remained unattacked, the fluoroindate glasses tested seem to be relatively durable for practical use.

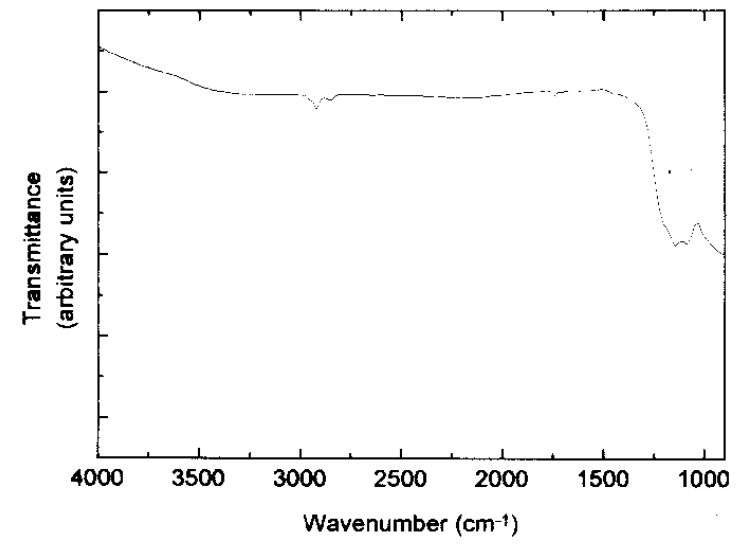

Figure 3. IR spectrum of $\mathrm{InF}_{3}$ glass after water attack.

Simmons et $\mathrm{al}^{9}$ report that the $1640 \mathrm{~cm}^{-1}$ band, in the case of water leaching of fluorozirconate glasses, come from oxide complexes which arise upon dehydration of samples whose surfaces were severely attacked by water. The small peak around $1000 \mathrm{~cm}^{-1}$ is probably due to contamination of $\left(\mathrm{SO}_{4}\right)^{2-}$ ions from $\mathrm{KHSO}_{4}$ used for cleaning the platinum crucible in which the glasses were melted.

\section{CONCLUSION}

Under the testing conditions imposed, the samples of fluoroindate glasses tested exhibited a higher resistance to moisture attack than the fluorozirconate glasses. The incorporation of magnesium in the system studied seems to provide glasses with a good chemical durability against water corrosion, particularly the samples in the In-Ba-Zn-Sr system. In addition it was observed that corrosion in the fluoroindate glasses investigated was limited to a thin surface layer. A systematic study of the effects of an aqueous medium on the chemical durability of magnesium containing fluoride glasses is necessary to confirm and to clearly quantify these assessments, as well as to define the possible mechanisms of water corrosion and the rate of this process.

\section{REFERENCES}

1. Poulain, M.; J. Non-Cryst. Sol. 1995, 184, 103.

2. Costa, B. J. and Messaddeq, Y. ; Anais $40^{\circ}$ Congr. Bras. Ceram. 1996.

3. Poulain, M. ; Poulain, M.; J. Non-Cryst. Sol. 1992, $140,87$.

4. Soufiane, A.; Ph.D. Thesis, 1992, Universite de Rennes I, Rennes, France.

5. Frischat, G. H. ; Overbeck, I.; J. Amer. Ceram. Soc. 1984, 67, C-238.

6. da Silva, D. V.; M.Sc. Dissertation, 1996, I.Q. - UNESP, Araraquara, Brazil.

7. da Silva, D. V. ; Proc. Intern. Symp. On Glass Cryst., 1996, Florianopolis, Nov. 4-8., Brazil.

8. Colthup, N. B.; J. Opt. Soc. Am. 1950, 40, 397.

9. Simmons, C. J.; Sutter, H.; Simmons, J. H.; Tran, D. C.; Mater. Res. Bull. 1982, 17, 1203. 